Original research article

\title{
Staphylococcus aureus carrier state among surgery and orthopedics health care personnel of a tertiary care center
}

\author{
Sara Varghese, Devraj Ramakrishnan, Jyothi Rajahamsan, \\ Sreekanth Karthikeyan Balakrishnan, Indu Rajendradas Mercybai, \\ Mathew Joseph Valamparampil*, Ananth Mohan, Amjith Rajeevan \\ Government Medical College, Thiruvananthapuram, Kerala, India
}

\section{A R T I C L E I N F O}

\section{Article history:}

Received 11 September 2017

Accepted 21 October 2017

Available online 23 October 2017

\section{Keywords:}

Staphylococcus aureus

Healthcare

MRSA

\begin{abstract}
A B S T R A C T
Introduction: The objective of this study was to estimate the proportion of Staphylococcus aureus carriers among the health care personnel in General Surgery and Orthopaedics Departments of Government Medical College, Thiruvananthapuram and also to estimate the proportion of methicillin resistant strains of Staphylococcus aureus (MRSA) among them.

Methods: All the health care personnel in General Surgery and Orthopaedics Departments of Government Medical College, Thiruvananthapuram, who were willing to take part in the study and gave consent were recruited. A semi structured questionnaire was used for data collection. Nasal swabs from both nostrils and hand impression specimens from both hands were obtained and cultured on blood agar and MacConkey agar plates for detection of Staphylococcus aureus, followed by antibiotic sensitivity testing. MRSA was confirmed with Hichrome agar.

Results: Out of the 341 subjects, 125 (36.7\%) tested positive for Staphylococcus aureus, and 10 (2.93\%) tested positive for MRSA. All 10 study participants were from the surgery department.

Conclusion: More than one-third of the healthcare personnel were found to be carriers of Staphylococcus aureus. The prevalence of $2.93 \%$ of healthcare personnel being carriers of MRSA is a cause of concern. The causes of personnel from only one department being found to be carriers, need to be evaluated further for finding the determinants of such carrier state.
\end{abstract}

(c) 2017 INDIACLEN. Published by Elsevier, a division of RELX India, Pvt. Ltd. All rights reserved.

\section{Introduction}

Hospital acquired infections or nosocomial infections are defined as infections acquired during hospital care which are not present or incubating at the time of admission. These are cross infections of one patient by another or by doctors, nurses and other hospital staff, while in hospital. ${ }^{1}$ The factors such as low level of immunity among the hospitalized patients, high number of invasive examinations and treatment, poor patient care practices and hospital environment may contribute to the spread of infections among patients. An increase in the incidence of nosocomial infection shows a poor quality of health care delivery. The development of antibiotic resistance is a new challenge, which calls for a re-alignment of our infection control strategy. ${ }^{1,2}$ The role of Staphylococcus aureus as a

\footnotetext{
* Corresponding author at: Department of Community Medicine, Government Medical College, Thiruvananthapuram, Kerala, India.

E-mail address: mathewjvalamparampil@gmail.com (M.J. Valamparampil).
}

persistent nosocomial and community acquired pathogen has become one of the major global health concerns. ${ }^{3}$

The incidence of Health care associated infections (HCAIs) in the ICU was estimated to be $16 \%$ in a study done in Goa, half of which were contributed by ventilator associated pneumonia, followed by urinary tract infection (35.6\%). Total one year cost of antibiotic therapy for HCAIs in this tertiary care ICU was estimated to be approximately Rs. 2 million (US $\$ 32,000$ ) and the mean cost of antibiotics per HCAI to be Rs. 17,000 (US\$255). Failure of prevention of HCAIs in the ICU thus puts a significant economic burden on the patient and the healthcare network. ${ }^{4}$ In a study done in six cities of India, the proportion of surgical site infections was estimated to be $4.2 \% .^{5}$ Central line associated blood stream infections were also found out to be an important HCAI. ${ }^{6}$

Staphylococci have a history of developing resistance quickly and successfully to antibiotics. The rapidity with which resistance emerges and its extent are proportional to the intensity of antimicrobial use. Resistance first emerges in populations with a high frequency of infection, due to either underlying patient status or interventions compromising host defences, resulting in a high 
rate of antimicrobial use. Resistance to antibiotics are most commonly seen in acute health care facilities and areas such as intensive care unit, thus serving as a focal point in the emergence of anti-microbial resistance. ${ }^{7}$

Increase in MRSA is an issue of great public health significance and a heavy burden on country's economy. It has been proven that compared to methicillin sensitive Staphylococcus aureus, MRSA has a higher mortality rate which can be due to the lesser efficacy of vancomycin used for MRSA compared to the beta-lactams used for MSSA, and also to the longer time until an appropriate therapy is started for the MRSA patient. ${ }^{8}$ Treatment options are limited for MRSA patients as they are resistant to multiple drugs. MRSA infections in Canadian hospitals shows an increase from 0.46 to 5.90 per 1000 admissions between 1995 and 2004. Prolonged hospitalization of an average of 26 days of isolation per patient was required for patients harboring MRSA along with costly treatments and extensive surveillance and special control measures. The mean cost per MRSA patient was estimated to be $\$ 12,216$, with hospitalization expense amounting to $81 \%$ of the total cost. ${ }^{8}$

Five distinct tools have been identified by the health care facilities in developed countries for active MRSA surveillance of high risk patients. They are Patient empowerment, Barrier precautions, Hand hygiene, Disinfection and Cleaning. ${ }^{9}$

Identification of patients and healthcare workers colonized with MRSA, combined with hand hygiene and other precautions have been shown to be effective in reducing the transmission and controlling the spread of MRSA. ${ }^{10}$ The most cost-effective way to lower HCAIs and their related adverse outcomes was found to be the standardization of infection control training and practices in a study done in a cardiovascular surgical unit. ${ }^{6}$

Numerous studies across the world have proven that Asymptomatically colonized patients and health care workers are the major sources of methicillin-resistant Staphylococcus aureus (MRSA) in the hospital environment, health care workers being more commonly identified as links in the transmission of MRSA between patients. ${ }^{10-14}$ In this context, it is important to screen the health care personnel for Staphylococcus aureus carrier state so that the incidence of nosocomial infections among patients can be reduced. This helps in creating an evidence base, thus paving the way for effective infection control programs. The objective of this study was to estimate the proportion of Staphylococcus aureus carriers among the health personnel in General Surgery and Orthopedics Departments of Government Medical College, Thiruvananthapuram, a tertiary care center in the state of Kerala in India, and also to estimate the proportion of methicillin resistant strains of Staphylococcus aureus among them.

\section{Methods}

The design was a hospital based descriptive study. Study setting was General Surgery and Orthopedics departments of Government Medical College, Thiruvananthapuram. The departments of General Surgery and Orthopedics were chosen because of the high patient load and the risk of post-operative infections involved. Subsequent expansion of this study to the entire hospital is under discussion. Study participants were staff doctors, post graduate doctors, interns, staff nurses and nursing assistants from wards, outpatient units and operation theatres of general surgery and orthopedics. Total staff strength of Departments of Surgery and Orthopedics was 365 and all of them were requested to take part in the study. Twenty four healthcare staff who did not give consent was excluded. Study period was from August 2015 to April 2016. Doctors doing internship in various units of both departments were enrolled in the study. Clearance was obtained from the Human Ethics Committee of Government Medical College Thiruvananthapuram. Heads of the department of General Surgery and
Orthopedics were briefed about the study and permissions were obtained.

The complete procedure was explained to all subjects and their informed written consents were obtained. Then they were interviewed and the interview schedules were filled by the researchers. Hand impression specimens were taken from both hands first on blood agar plates, followed by MacConkey agar plates, one set for each hand. The subjects were asked to just touch the pulp of all five fingers on the plates and the covers were put back immediately on the plates for preventing environmental contamination. Sterile cotton swabs were used to collect the nasal swabs from the anterior nares. The tip of a $15 \mathrm{~cm}$ sterile cotton swab was inserted, one in each nostril and rotated two times over the nares and taken out of the nose. It was replaced immediately into its screw-capped sterile bottle. The specimens were transported to the microbiology laboratory immediately and processed. $^{15}$

Specimens were cultured on blood agar and MacConkey agar. Culture plates were incubated overnight at $37^{\circ} \mathrm{C}$. The isolates were subjected to following biochemical tests - Catalase, Coagulase (Slide and Tube), Urea Hydrolysis, Mannitol Fermentation, Oxidation Fermentation. ${ }^{16}$ Antibiotic sensitivity testing was done by Kirby Bauer method in Mueller Hinton Agar plate by using cefoxitin $30 \mu \mathrm{g}$ disc. Test strains with zone diameter less than $22 \mathrm{~mm}$ were taken as resistant. These strains were cultured on $\mathrm{Hi}$ Chrome agar for confirmation of MRSA. On Hi Chrome agar, spontaneous green coloration of $\alpha$ glucosidase producing colonies obtained in the presence of cefoxitin $(4 \mathrm{mg} / \mathrm{dl})$ were considered positive. $^{15}$

\section{Results}

A survey was conducted and Nasal swabs and hand impressions on blood and MacConkey agar plates of 341 health care staff from surgery and orthopedics departments of medical college, Thiruvananthapuram were analyzed for the presence of Staphylococcus aureus and methicillin resistance.

The mean age of the study participants was 34.50 (10.89). Males comprised of $45.5 \%$ of the study subjects. Out of the study subjects, $72.7 \%$ had educational qualification of graduation or higher. Doctors (including interns) comprised of $51.6 \%$ of the study population, staff nurses $29.9 \%$ and the rest were nursing assistants and grade 2 staff. Personnel from department of General Surgery were 231 (67.7\%) while from Orthopedics were 103 (30.2\%). Seven (2.1\%) were dedicated operation theatre personnel. History of frequent skin or throat infections were given by $22 \%$ of the study participants.

Out of the 341 subjects, 125 (36.7\%) tested positive for Staphylococcus aureus. They fall into three groups; 86 (25.2\%) tested positive in both nasal swab and hand impression specimen, $22(6.5 \%)$ tested positive only in hand impression specimen whereas 17 (5\%) tested positive only in nasal swab (Table 1 ).

Only 10 out of 341 (2.93\%) tested positive for MRSA. All 10 study participants were from the surgery department. Those who tested positive for hand impression alone was $6(1.76 \%)$ and for nasal swab alone was $4(1.2 \%)$. None of the subjects tested positive in both nasal swab and hand impression. Axillary swabs also yielded

Table 1

Staphylococcal positivity.

\begin{tabular}{ll}
\hline Types of Staphylococcus Positive specimen & Frequency $(\mathrm{n}=341)$ \\
\hline Any specimen positive & $125(36.7 \%)$ \\
Both Nasal Swab and Hand impression positive & $86(25.2 \%)$ \\
Only Hand impression positive & $22(6.5 \%)$ \\
Only Nasal swab positive & $17(5 \%)$ \\
\hline
\end{tabular}


negative results. Sensitivity pattern was done according to Clinical \& Laboratory Standards Institute (CLSI) guidelines and all MRSA samples were found to be highly resistant to Gentamycin, Septran and Erythromycin. All samples were found to be sensitive only for Vancomycin and Linezolid and the Minimum Inhibitory Concentration (MIC) of Vancomycin was also calculated as per CLSI guidelines. No genetic analysis could be done in this study for characterization of MRSA isolates. Only $73 \%$ of the study participants said that soap and water were always available. Of the 341 participants, 326 (95.6\%) mentioned washing as their usual method of cleaning hands and $282(82.7 \%)$ said that they regularly use soap for washing hands. A large majority, 303 participants (88.9\%) said that hand sanitisers are not usually available to them. Further follow-up was advised at the Infectious Disease Unit of the institution.

\section{Discussion}

The proportion of Staphylococcus aureus carrier state among 341 health care personnel of surgery and orthopedics department of medical college, Thiruvananthapuram was estimated as $36.7 \%$ and that of MRSA as 2.93\%, MRSA forming 7.98\% of total staphylococcus isolates. No such large scale study has been reported from southern part of India. In a study conducted among 316 healthcare workers at a Tertiary Care Hospital in Assam, Staphylococcus aureus was isolated in $22.2 \%$ and the carrier rate was the highest in the orthopedics department, followed by those in the surgery and the gynecology departments. Methicillin resistance was seen in $11.43 \%$ of the Staphylococcus aureus isolates; $2.53 \%$ of the whole. ${ }^{17}$ In another study done in tertiary care hospital in south India, only $1.8 \%$ health care workers were found to have methicillin-resistant Staphylococcus aureus (MRSA) carrier state. ${ }^{11}$ A 5 year study of MRSA in Netherlands determined that $2.1 \%$ of 8,403 patients and $0.2 \%$ of 13,195 health care workers were MRSA carriers. The average number of nosocomial transmissions was 6.7 per year. ${ }^{18}$ In 2004 a study done on the carriage of MRSA among hospital employees, sixty MRSA carriers were identified among 965 healthcare providers, giving a proportion of $6.2 \%$ which was higher among staff from clinical wards than from elsewhere $(9.0 \%$ compared to $2.1 \%){ }^{19}$ A study on the bacterial contamination of health care workers' white coats in 2009 revealed that of the 149 white coats, 34 (23\%) were contaminated with Staphylococcus aureus, amongst which $6(4 \%)$ were MRSA. Staphylococcus aureus contamination was more prevalent in residents and those working in inpatient settings. $^{20}$

A study done in Los Angeles on 65 subjects relating to the body colonisation of community associated MRSA revealed that among $37 \%(24 / 65)$ who were found to be MRSA colonized, $25 \%$ of patients $(16 / 65)$ were colonized in the nares, $6 \%(4 / 65)$ in the axilla, $17 \%(11 /$ $64)$ in the inguinal area, and 13\% (7/54) in the rectal area. Thus it can be inferred that nares are the most common site of MRSA colonisation. ${ }^{21}$ In our study, 6 out of 10 were positive for hand impressions and only 4 out of 10 were nasal carriers of MRSA. There were no axillary carriers. Inguinal and rectal swabs were not taken in our study. The findings related to site of colonisation is seen to be different in this study when compared to western studies.

In our study, $95.6 \%$ of the health care personnel resorted to hand washing for keeping their hands clean. Whereas $82 \%$ regularly used soap for hand washing, the continuous availability of soap and water was reported by only $73 \%$. A study in Vietnam concludes that the combination of simple infection control measures and appropriate use of antibiotics decrease the prevalence of MRSA. ${ }^{22}$ The role of barrier techniques and infection control in reducing the prevalence of MRSA has been elicited in different studies. ${ }^{23,24}$ We regret that we did not collect details of the exact time of last hand wash. This was because such a variable will be extremely subjective as we cant ascertain the quality of the hand washing done before sample collection. Hence we did not try to find association between a definite variable like presence of Staphylococcus aureus and a subjective variable like time of washing hands. Analysis for finding association of carriers of Staphylococcus aureus and MRSA were not done. Being a carrier for Staphylococcus aureus is protective against MRSA carrier state, while the actual number of 10 MRSA carriers found from this study is inadequate for performing any statistical analysis. The participants found positive for MRSA were provided with $2 \%$ mupirocin ointment, were taught about the method of application and advised treatment for 1 week duration

The methicillin susceptible Staphylococcus aureus colonization may be protective against colonization by MRSA. ${ }^{25}$ Hence we focussed specifically on MRSA. As the number of MRSA positive cases identified were just 10 , the non-participation by 24 health care personnel might have an influence on the results.

Proportion of MRSA was estimated to be $2.93 \%$ among the surgery and orthopedics health care personnel of government medical college, Thiruvananthapuram. All MRSA positives were detected from the surgery department. With the hospital protocol regarding to infection control being equally applicable to both departments, the reasons for carrier state among only one surgical department staff need to be investigated more thoroughly. The role of chronic infections like diabetic ulcers being a continued source of MRSA need to be evaluated. Of all the study participants, almost all (95.6\%) resorted to hand washing for keeping their hands clean, whereas only $82 \%$ regularly used soap for hand washing but only $73 \%$ of the study participants said that soap and water were always available. Hence soap and water must be made available all the time and all the health care staff should be advised to regularly use soap for washing hands. Use of alcohol-based hand rub have been prescribed by WHO for healthcare personnel before/after touching patients or devices and even after removing gloves. Such practices need to be adapted by healthcare professionals to combat hospitalacquired infections of drug-resistant pathogens. Periodic screening for Staphylococcus aureus carrier state and methicillin resistance is recommended in future.

\section{Source of Funding}

State Board of Medical Research, Government of Kerala. Order Number A2-SBMR (2013-2014)/15790/2013/MCT dated 19/2/2015

\section{Conflicts of Interest}

Authors declare no conflicts of interest

\section{References}

1. General Information About MRSA in Healthcare Settings [Internet]. Centers for Disease Control and Prevention; 2017. [cited 2016 Oct 24]. Available from: http://www.cdc.gov/mrsa/healthcare/index.html.

2. Health Care-Associated Infections Fact Sheet WHO [Internet]. . [cited 2016 Oct 24]. Available from: http://www.who.int/gpsc/country_work/ gpsc_ccisc_fact_sheet_en.pdf.

3. Nwankwo EO, Nasiru MS. Antibiotic sensitivity pattern of Staphylococcus aureus from clinical isolates in a tertiary health institution in Kano, Northwestern Nigeria. Pan Afr Med J. 2011;8:4.

4. Misal DD, Maulingkar SV, Bhonsle S. Economic burden of antibiotic treatment of healthcare-associated infections at a tertiary care hospital ICU in Goa. India Trop Doct. 2016;47(3):197-201.

5. Singh S, Chakravarthy M, Rosenthal VD, et al. Surgical site infection rates in six cities of India: findings of the International Nosocomial Infection Control Consortium (INICC). Int Health. 2015;7(September (5)):354-359.

6. Singh S, Kumar RK, Sundaram KR, Kanjilal B, Nair P. Improving outcomes and reducing costs by modular training in infection control in a resource-limited setting. Int J Qual Health Care J Int Soc Qual Health Care. 2012;24(December (6)):641-648. 
7. Nicolle L. Infection control programmes to contain antimicrobial resistance [Internet]. World Health Organisation; 2001. Available from: http://www.who. int/csr/resources/publications/drugresist/infection_control.pdf.

8. Goetghebeur M, Landry P-A, Han D, Vicente C. Methicillin-resistant Staphylococcus aureus: a public health issue with economic consequences. Can J Infect Dis Med Microbiol. 2007;18(January (1)):27-34.

9. Perlin JB, Hickok JD, Septimus EJ, Moody JA, Englebright JD, Bracken RM. A bundled approach to reduce methicillin-resistant Staphylococcus aureus infections in a system of community hospitals. J Healthc Qual Off Publ Natl Assoc Healthc Qual. 2013;35(June (3))57-68 quiz 68-69.

10. Barnes SL, Morgan DJ, Harris AD, Carling PC, Thom KA. Preventing the transmission of multidrug-resistant organisms (MDROs): modeling the relative importance of hand hygiene and environmental cleaning interventions. Infect Control Hosp Epidemiol Off J Soc Hosp Epidemiol Am. 2014;35(September (9)):1156-1162.

11. Mathanraj S, Sujatha S, Sivasangeetha K, Parija SC. Screening for methicillinresistant Staphylococcus aureus carriers among patients and health care workers of a tertiary care hospital in south India. Indian J Med Microbiol. 2009;27(January (1)):62.

12. Bilavsky E, Lerman Y, Salomon J, et al. Carriage of methicillin-resistant Staphylococcus aureus on admission to European rehabilitation centres-a prospective study. Clin Microbiol Infect. 2012;18(June (6)):E164-E169.

13. Thompson Jm, Gündoğdu A, Stratton Hm, Katouli M. Antibiotic resistant Staphylococcus aureus in hospital wastewaters and sewage treatment plants with special reference to methicillin-resistant Staphylococcus aureus (MRSA). J Appl Microbiol. 2013;114(January (1)):44-54.

14. Shibabaw A, Abebe T, Mihret A. Antimicrobial susceptibility pattern of nasal Staphylococcus aureus among Dessie Referral Hospital health care workers, Dessie, Northeast Ethiopia. Int J Infect Dis IJID Off Publ Int Soc Infect Dis. 2014;25 (August):22-25.

15. Koneman EW. Koneman's Color Atlas and Textbook of Diagnostic Microbiology. Lippincott Williams \& Wilkins; 20061764 p.
16. Mackie TJ. Mackie McCartney Practical Medical Microbiology. Churchill Livingstone; 1996:1002.

17. Rongpharpi SR, Hazarika NK, Kalita H. The prevalence of nasal carriage of Staphylococcus aureus among healthcare workers at a tertiary care hospital in Assam with special reference to MRSA. J Clin Diagn Res JCDR. 2013;7(February (2)):257-260.

18. Vos MC, Behrendt MD, Melles DC, et al. 5 years of experience implementing a methicillin-resistant Staphylococcus aureus search and destroy policy at the largest university medical center in the Netherlands. Infect Control Hosp Epidemiol. 2009;30(October (10)):977-984.

19. Eveillard M, Martin Y, Hidri N, Boussougant Y, Joly-Guillou M-L. Carriage of methicillin-resistant Staphylococcus aureus among hospital employees: prevalence, duration, and transmission to households. Infect Control Hosp Epidemiol. 2004;25(February (2)):114-120.

20. Treakle AM, Thom KA, Furuno JP, Strauss SM, Harris AD, Perencevich EN. Bacterial contamination of health care workers' white coats. Am J Infect Control. 2009;37(March (2)):101-105.

21. Yang ES, Tan J, Eells S, Rieg G, Tagudar G, Miller LG. Body site colonization in patients with community-associated methicillin-resistant Staphylococcus aureus and other types of S. aureus skin infections. Clin Microbiol Infect. 2010;16(May (5)):425-431.

22. Schultsz C, Bootsma MCJ, Loan HT, et al. Effects of infection control measures on acquisition of five antimicrobial drug-resistant microorganisms in a tetanus intensive care unit in Vietnam. Intensive Care Med. 2013;39(April (4)):661-671.

23. Lee BY, Bailey RR, Smith KJ, et al. Universal methicillin-resistant Staphylococcus aureus (MRSA) surveillance for adults at hospital admission: an economic model and analysis. Infect Control Hosp Epidemiol. 2010;31(June (6)):598-606.

24. Dziekan G, Hahn A, Thüne K, et al. Methicillin-resistant Staphylococcus aureus in a teaching hospital: investigation of nosocomial transmission using a matched case-control study. J Hosp Infect. 2000;46(December (4)):263-270.

25. Stevens AM, Hennessy T, Baggett HC, Bruden D, Parks D, Klejka J. Methicillinresistant Staphylococcus aureus carriage and risk factors for skin infections, southwestern Alaska, USA. Emerg Infect Dis. 2010;16(May (5)):797-803. 